\title{
ECOCARDIOGRAFÍA EN REPOSO E INCAPACIDAD LABORAL
}

\author{
DOMINGO SÁNCHEZ M.
}

Médico Inspector de la Administración de la Seguridad Social. D.P. INSS en Sevilla.

\begin{abstract}
AGRADECIMIENTOS
A la Dra. Ruiz Figueroa, por su inestimable ayuda y participación en la revisión de este trabajo. A los compañeros (Médicos Inspectores del INSS) que aportaron voluntariamente sus casos a la serie recopilada.
\end{abstract}

\section{AUTORIZACIONES}

La publicación de este trabajo cuenta con la autorización de la Dirección Provincial del INSS de Sevilla.

\section{RESUMEN}

El método empírico sigue siendo el procedimiento de estimación del grado de incapacidad laboral, reforzado con la interpretación de los datos clínicos y pruebas complementarias. Para los trabajadores con secuelas de patología cardiaca, la ecocardiografía en reposo es la prueba complementaria más usada para objetivar deficiencias orgánicas e inferir limitaciones funcionales, tanto por su accesibilidad como por su especificidad, bajo coste, fácil acceso y nulo riesgo. Habitualmente, en la revisión de trabajadores calificados como incapacitados permanentes, confirmamos que realizan sin limitación significativa una actividad laboral con igual o mayor requerimiento físico que aquella para la que se le consideró incapacitado años atrás. Consideramos necesario el análisis de los fundamentos de epidemiología clínica aplicada a la toma de decisiones en medicina evaluadora, para reflexionar sobre el valor del soporte científico de nuestras decisiones en medicina aplicada a la incapacidad laboral. Se expone un estudio descriptivo de una serie de 100 casos en los que la ecocardiografía fue considerada como prueba básica para la determinación del grado funcional por el Médico Evaluador. Se exponen una serie de reflexiones sobre los resultados, y, sobre el estudio de la literatura especializada aplicable al análisis crítico de los resultados.

\section{PALABRAS CLAVES}

Incapacidad laboral, Grado funcional, ecocardiografía, medicina evaluadora. 


\section{INTRODUCCIÓN}

\section{Antecedentes y justificación del estudio}

Actualmente, el método empírico sigue siendo el procedimiento de estimación del grado de incapacidad laboral, aunque reforzado con la aportación científica que da la interpretación de los datos clíni$\cos$ y los hallazgos en pruebas complementarias $(1,3)$.

Sin embargo, la necesidad de contrastar para objetivar (10) el sustrato patológico de las alegaciones subjetivas, realizadas por los trabajadores sometidos a evaluación de su incapacidad laboral, requiere el uso de pruebas complementarias que ayuden a cuantificar las deficiencias presentes, y de ellas inferir el grado de limitación funcional.

Para la actividad evaluadora de las Unidades Médicas (5) de los Equipos de Valoración de Incapacidades del INSS (UMEVI) se utilizan habitualmente las pruebas diagnósticas de aplicación habitual en la práctica clínica ; tanto más cuanto más cuantificables son sus resultados.

De estos resultados se infieren niveles de afectación funcional de los órganos, aparatos o sistemas, que traducimos de forma empírica a grados de deficiencia, artificialmente clasificados en niveles o grados funcionales (4).

En los procesos para los que podemos obtener este tipo de resultados, las pruebas diagnósticas tienen un peso importante en la calificación final de la incapacidad, en muchas ocasiones determinante o decisivo.

En los trabajadores con patología cardiaca, la ecocardiografía en reposo es la prueba complementaria más usada para apoyar las deficiencias e inferir limitaciones, tanto por su accesibilidad como por su especificidad, bajo coste, fácil acceso y nulo riesgo.

Los excelentes resultados publicados en las dos últimas décadas sobre AVAC (años de vida ajustados a calidad) y AVAD (años de vida ajustados a discapacidad) (9) -para los procedimientos de la medicina contemporánea y muy especialmente tras los avances en Cardiología-, no parecen asociarse (al menos de forma intuitiva) a una reducción notoria de las calificaciones de Incapacidad Laboral derivada de patología cardiaca.

De forma habitual, revisamos la situación de trabajadores calificados como incapacitados permanen- tes para una determinada actividad (calificación fundamentada en la situación clínico-funcional y los datos más o menos objetivos de una o varias pruebas diagnósticas). Sin embargo, en numerosas ocasiones, confirmamos que dicho trabajador realiza sin limitación significativa una actividad laboral de igual o más nivel de requerimiento físico que aquella para la que se le calificó incapacitado uno o más años atrás (aún sin modificación significativa en los resultados de las pruebas, una vez actualizadas, ni en evolución clínica documentada).

Frente a la realidad, anteriormente expuesta, consideramos necesario el análisis de los fundamentos de epidemiología clínica aplicada a la toma de decisiones en medicina evaluadora, para reflexionar sobre el valor del soporte científico de nuestras decisiones en medicina aplicada a la incapacidad laboral.

Sin embargo, de plantearse la necesidad de este análisis, se podría llegar a determinar la validez, o no, de la aplicación de las distintas pruebas diagnósticas (habituales en clínica) a la calificación de la incapacidad laboral; lo que no hemos hallado en las publicaciones especializadas de la literatura médica, hasta la fecha. Igualmente, se podría deducir el coste-beneficio y el coste-utilidad de las pruebas más usadas para aproximarse, de manera más objetiva, al grado de incapacidad con métodos eficaces, eficientes y validados en el ámbito evaluador.

\section{Hipótesis de trabajo y pertinencia del estudio (1 a 10):}

La Ley General de la Seguridad Social (LGSS), que determina el concepto de Incapacidad Permanente y sus grados según la repercusión funcional, limita de forma expresa el carácter de las reducciones anatómicas o funcionales, que deberán ser "graves" y "susceptibles de determinación objetiva" tal que "disminuyan o anulen su capacidad laboral" (y sean "previsiblemente definitivas").

Así, la calificación de incapacidad laboral permanente (IP) está determinada legalmente en varios grados, pero son la Incapacidad Permanente Parcial (IPP) y la Incapacidad Permanente Total (IPT) las más problemáticas de fundamentar en Medicina Evaluadora, por estar referidas de forma expresa a la actividad laboral propia del asegurado (la cual puede tener niveles de requerimiento físico y/o psíquico muy diversos, técnicamente muy difíciles de medir, a veces).

Las pruebas complementarias de uso habitual en Medicina Evaluadora, están validadas en el ámbito 
clínico y tienen utilidad probada en el proceso diagnóstico. Centrándonos en la evaluación de las deficiencias de origen cardiológico, las guías de uso habitual para la graduación del menoscabo permanente según la bibliografía, se basan de forma muy preferente en las pruebas complementarias de mayor acceso y disponibilidad en la práctica clínica (principalmente ecocardiografía, prueba de esfuerzo y de medicina nuclear).

Con excepción de las pruebas de esfuerzo (aún con sus muchas limitaciones, y, en sus diferentes modalidades; ver Tablas homologadas por la OMS de Capacidad Funcional en METS requerida para distintas actividades), no hemos hallado en la revisión bibliográfica realizada a propósito del presente trabajo, estudios que demuestren un grado significativo de asociación entre un determinado resultado en una prueba diagnóstica y el grado de incapacidad para la realización de un nivel de trabajo físico determinado.

Pensamos que el análisis crítico de este escenario teórico podría llevar al diseño de estudios prospectivos que permitieran establecer, o al menos aproximarse, a los diversos grados funcionales y las indicaciones para las distintas pruebas complementarias en medicina evaluadora.

La justa distribución de las prestaciones y recursos públicos, según las necesidades de los asegurados en nuestro Sistema de Seguridad Social, lleva al planteamiento de análisis coste-beneficio (ACB), coste-efectividad (ACE) y coste-utilidad (ACU), que en el ámbito clínico-asistencial están necesariamente asumidos desde hace varias décadas. La selección de las pruebas complementarias y sus parámetros de función (indicadores precisos) nos parece fundamental para ajustar las calificaciones de IP a las limitaciones funcionales reales de los trabajadores demandantes de prestaciones por incapacidad. De aquí surge la hipótesis de trabajo y la justificación de la investigación planteada en el presente trabajo.

\section{OBJETIVOS}

Con este trabajo pretendemos alcanzar los siguientes objetivos:

A) General: Reflexionar sobre el papel que está jugando el uso de las pruebas complementarias al diagnóstico, en la valoración de la incapacidad laboral.
B) Específicos:

- Dirigir la reflexión a la calificación del grado de incapacidad, tras el estudio con ecocardiografía en reposo, de las deficiencias y limitaciones por patología cardiaca.

- Describir una serie de 100 casos recopilados en una UMEVI, durante 6 meses (octubre-06/ abril-07), y, en los que la principal deficiencia orgánica y/o funcional era cardiológica.

C) Secundario: Llegado el caso, fundamentar el inicio de un estudio prospectivo para evaluar la capacidad funcional efectiva en trabajadores con y sin calificación de incapacidad laboral, y su relación o no con los resultados de las pruebas diagnósticas utilizadas en el procedimiento evaluador.

\section{MÉTODO}

Estudio en profundidad de un caso, comentado y discutido en sesión clínica de la UMEVI. Se consideró de especial interés por la mayor discrepancia entre pruebas y el valor teórico (probabilidad previa) de la ecocardiografía en reposo para la miocardiopatía presentada por la trabajadora:

Análisis de una serie de 100 casos consecutivos correspondientes a expedientes evaluados en una UMEVI (durante los meses de octubre-06 a abril-07) con las siguientes condiciones de inclusión:

A) $\mathrm{Su}$ comunicación fue voluntaria por los Médicos de la Unidad.

B) Tenían como origen de la deficiencia principal una patología cardiaca (C. Isquémica, C. Hipertensiva, Miocardiopatía, Valvulopatía).

C) La ecocardiografía reciente (en los últimos 6 meses) sería prueba complementaria básica (a criterio del Médico Evaluador) para valorar la deficiencia principal reflejada en el Informe Médico de Síntesis (IMS).

D) El Equipo de Valoración de Incapacidades (EVI) no conocería, en el momento de la calificación del grado de incapacidad, que el caso corresponde a esta serie (simple ciego).

\section{Revisión bibliográfica ${ }^{1}$ acerca de:}

A) los rangos (normalidad-patológicos) de los parámetros ecocardiográficos y de su posible aplicación a los criterios de aptitud física para el trabajo, así como de la sensibilidad y especificidad de los

Será objeto de próxima publicación 
parámetros obtenidos mediante ecocardiografía en reposo.

B) los criterios de aplicación de la ecocardiografía (en sus diferentes modalidades, y en relación con otras pruebas de imagen), referidos en la literatura actual especializada, con potencial aplicación a la determinación del grado de afectación de la función cardiaca.

C) los requerimientos cardiacos en el trabajo con carga física, con traducción en los parámetros de uso habitual en clínica.

\section{RESULTADOS}

Estudio en profundidad de un caso:

- Da C.D.G., trabajadora española de 49 años, de profesión ATS / DUE con destino en consultas de un Centro de Especialidades Periférico del Servicio Público de Salud (SPS)

- Inicia proceso de I.T. por E.C. en el mes de abril-2005, por astenia y mal estado general con disnea en reposo (inicialmente diagnosticada de Trastorno de Ansiedad Generalizada e iniciándose tratamiento Psiquiátrico que fracasa). Al aparecer edemas maleolares y pérdida ponderal de $20 \mathrm{Kg}$, se realizan valoraciones y estudios cardiológico, llegándose al diagnostico de Miocardiopatía Dilatada de causa desconocida. Igualmente se diagnostica diabetes mellitus (DM) tipo 2 no complicada.

- En los estudios cardiológicos de junio-05 se confirman: insuficiencia cardiaca congestiva con ecocardiografía patológica (Fracción de eyección $\mathrm{FE}=15 \%$, hipertensión pulmonar moderada y regurgitación mitral).

- Mejora con tratamiento farmacológico y se estabiliza en grado funcional cardiaco II/IV, mejorando la FE en septiembre-05 hasta 33\% con tensión arterial $\mathrm{TA}=120 / 80$, sin edemas.

- Transcurrido, aproximadamente, un año de incapacidad temporal (I.T.) (mayo-06), el SPS realiza Propuesta de I.P. con el diagnostico de: "Miocardiopatía Dilatada de origen idiopático, sin poder descartar Miocarditis, con $\mathrm{FE}=33 \%$ (en ecocardiografía de septiembre2005) y DM tipo 2".

- En dicha propuesta se manifiesta lo siguiente:
"La consideramos incapacitada de forma permanente para el desarrollo de tareas laborales que requieran de manipulación de cargas, posturas forzadas y mantenidas, moderadosmínimos esfuerzos, cambios bruscos de temperatura y situaciones estresantes".

- No se aportan nuevos datos de significación respecto a lo descrito en la evolución de septiembre-2005.

- Se solicita y recopila la información clínicoasistencial del SPS, desde la fecha de la propuesta de IP al momento de la evaluación en UMEVI, consistente en :

- Notas evolutivas en revisiones cardiológicas: Oligosintomática, salvo astenia atribuida por el clínico a medicación ( beta-bloqueantes y diuréticos), hasta octubre-2006 en que se aplaza ya a 6 meses el siguiente control cardiológico por buena evolución clínica.

- Ventriculografía septiembre-2005: FE= $33,6 \%$, V.I. dilatado $(70 \times 87 \mathrm{~mm})$, morfología globulosa, hipoquinesia severa septal, tardoquinesia anteroseptal.

- Se realizaron controles ecocardiográficos (Eco-Doppler de septiembre-2006, repetido en dos ocasiones por la discrepancia con la ventriculografía), que mostraron: estudio prácticamente normal, destacando un V.I. en los límites superiores de la normalidad $(\mathrm{DTDVI}=54 \mathrm{~mm})$, función sistólica normal con $\mathrm{FE}=65 \%$ a $73 \%$, función diastólica con patrón de alteración de la relajación del VI (como única anomalía, que no se observó al repetir la prueba a los 18 días), no signos de hipertensión pulmonar, ni alteraciones segmentarias de la contractilidad. A.I. $=38 \mathrm{~mm}$. Cavidades derechas y válvulas normales.

- Ventriculografías de octubre-2006: FE 63,8\%, dilatación del VI (56 x 64mm), morfología globulosa, hipoquinesia severa septal.

- En el momento de la evaluación por el ME del EVI, la asegurada alegaba cansancio -manifestado también en los controles asistenciales del año 2006-, y, disnea a medianos esfuerzos -no reflejada en dichos controles-.

- Se confirmó la buena evolución clínica en grupo funcional I-II, considerándole limitada 
para tareas con requerimientos físicos y/o de estrés, ambos en rango medio-alto.

- El Médico Evaluador (ME) propuso revisión a 1 año con reserva del puesto de trabajo. En el IMS se reflejaron todos los datos mencionados y se presentó en sesión EVI en Octubre-2006.

- La calificación emitida por el EVI fue de I.P. TOTAL para la profesión habitual (ATS / DUE en Centro Ambulatorio del SPS), con revisión a partir de octubre-2007.

\section{Descripción de una serie de casos:}

La calificación emitida por el EVI fue de I.P. TOTAL para la profesión habitual (ATS / DUE en Centro Ambulatorio del SPS), con revisión a partir de octubre-2007.

\begin{tabular}{|l|l|}
\hline Edad media & 54 a. \\
\hline Razón de sexos (H:M) & $2: 1$ \\
\hline
\end{tabular}

\begin{tabular}{|l|l|}
\hline Trabajo & $54 \%$ \\
\hline - Sedentario & $26 \%$ \\
\hline - Esfuerzo ligero & $11 \%$ \\
\hline - Esfuerzo medio & $9 \%$ \\
\hline - Esfuerzo alto &
\end{tabular}

\begin{tabular}{|l|l|}
\hline Diagnóstico: \\
\hline - C. Isquémica & $68 \%$ \\
\hline - C. HTA & $17 \%$ \\
\hline - Valvulopatía & $10 \%$ \\
\hline - Miocardiopatía & $5 \%$ \\
\hline
\end{tabular}

\begin{tabular}{|l|l|}
\hline Defecto ecocardiográfíco : Sistólico/ Diastólico \\
\hline No: & $71 \%$ \\
\hline SISTOLICO: & $9 \%$ \\
\hline DIASTOLICO: & $17 \%$ \\
\hline
\end{tabular}

\begin{tabular}{|l|l|}
\hline Alt. Motilidad Segmentaria & $\mathrm{SI}: 3 \%$ \\
\hline Dilatación A-V & $\mathrm{SI}: 4 \%$ \\
\hline $\mathrm{HVI}$ & $\mathrm{SI}: 13 \%$ \\
\hline
\end{tabular}

\begin{tabular}{|l|l|}
\hline Ergometría & $+(10 \%)$ \\
\hline Medicina Nuclear & $+(5 \%)$ \\
\hline
\end{tabular}

\begin{tabular}{|l|l|}
\hline \multicolumn{2}{|l|}{ Grupo Funcional Clínico (criterios Soc. Esp. Cardiol.) } \\
\hline I & $(71 \%)$ \\
\hline II & $(15 \%)$ \\
\hline III & $(14 \%)$ \\
\hline
\end{tabular}

\begin{tabular}{|l|l|}
\hline \multicolumn{2}{|l|}{ Grado Incapacidad.Permanente (según el EVI-INSS). } \\
\hline - NO I.P. & $25 \%$ \\
\hline - I.P.Parcial. & $2 \%$ \\
\hline - I.P.Total & $67 \%$ \\
\hline - I.P.A.bsoluta & $6 \%$ \\
\hline
\end{tabular}

El estudio de la asociación entre la variable cualitativa "Alteración predominante en Ecocardiografía en reposo" (Eco normal, Defecto sistólico, Defecto diastólico, Alteración de motilidad segmentaria) y la variable cualitativa "Incapacidad Permanente" (No IP, Si IP), obtenemos la siguiente tabla de contingencia con los valores de frecuencia absoluta observados:

\begin{tabular}{|l|l|l|l|l|l|}
\hline & $\begin{array}{l}\text { Eco } \\
\text { normal }\end{array}$ & $\begin{array}{l}\text { Defecto } \\
\text { sistólico }\end{array}$ & $\begin{array}{l}\text { Defecto } \\
\text { diastólico }\end{array}$ & $\begin{array}{l}\text { Alt.motilidad } \\
\text { segmentaria }\end{array}$ & Total \\
\hline No IP & 15 & 3 & 5 & 2 & 25 \\
\hline Si IP & 56 & 6 & 12 & 1 & 75 \\
\hline Total & 71 & 9 & 17 & 3 & 100 \\
\hline
\end{tabular}

Chi2 $=3.8557$

$\mathrm{Al}$ existir celdas con $<5$ casos los resultados pueden no ser representativos. Por ello, procedimos a agrupar las categorías de la variable "Alteración predominante en Ecocardiografía en reposo" en solo dos (Eco Normal, Eco alterada), obteniendo la siguiente tabla de contingencia con los valores de frecuencia absoluta observados:

\begin{tabular}{|l|l|l|l|}
\hline & Eco Normal & Eco alterada & Total \\
\hline No IP & 15 & 10 & 25 \\
\hline Si IP & 56 & 19 & 75 \\
\hline Total & 71 & 29 & 100 \\
\hline
\end{tabular}

\section{Chi2 $=1.3113 \quad$ Chi2 sin corregir $=1.9589$}

El estudio de la asociación entre la variable cualitativa "Grupo funcional estimado (GF)" (I, II, III) y la variable cualitativa "Calificación de IP por el EVI" (No IP, Si IP), obtenemos la siguiente tabla de contingencia con los valores de frecuencia absoluta observados:

\begin{tabular}{|l|l|l|l|l|}
\hline & GF I & GF II & GF III & Total \\
\hline No IP & 15 & 6 & 4 & 25 \\
\hline Si IP & 56 & 9 & 10 & 75 \\
\hline Total & 71 & 15 & 14 & 100 \\
\hline
\end{tabular}

Chi2 $=2.4633$

Al existir celdas con $<5$ casos puede no ser representativo.

En la determinación del GF, la ecocardiografía era un dato principal al que se le añaden los datos clínico-asistenciales recientes en la historia clínica del SPS y los obtenidos por el Médico Evaluador.

Para controlar la existencia de posibles asociaciones como origen de sesgos en el estudio, se descartó la existencia de asociación estadísticamente significativa entre el tipo de trabajo y el resultado de la ecocardiografía, así como entre el tipo de trabajo $\mathrm{y}$ el grado funcional. 
El estudio de la asociación entre la variable cualitativa "Grado de Incapacidad Permanente" (No IP, IPP, IPT, IPA) y la variable cualitativa "Tipo de Trabajo" (Sedentario, esfuerzo ligero, esfuerzo medio, esfuerzo alto), obtenemos la siguiente tabla de contingencia con los valores de frecuencia absoluta observados:

\begin{tabular}{|l|l|l|l|l|l|}
\hline & Sedentario & $\begin{array}{l}\text { esfuerzo } \\
\text { ligero }\end{array}$ & $\begin{array}{l}\text { esfuerzo } \\
\text { medio }\end{array}$ & $\begin{array}{l}\text { esfuerzo } \\
\text { alto }\end{array}$ & Total \\
\hline No IP & 15 & 1 & 6 & 3 & 25 \\
\hline IPP & 1 & 0 & 1 & 0 & 2 \\
\hline IPT & 38 & 18 & 8 & 3 & 67 \\
\hline IPA & 0 & 1 & 2 & 3 & 6 \\
\hline Total & 54 & 20 & 17 & 9 & 100 \\
\hline
\end{tabular}

Al existir varias celdas con ausencia de casos, procedimos a agrupar la variable "Grado de Incapacidad Permanente" en dos categorías (No IP, Si PP) y la variable "Tipo de Trabajo" en dos categorías (Sedentario-ligero, esfuerzo medio-alto), obteniendo la siguiente tabla de contingencia con los valores de frecuencia absoluta observados:

\begin{tabular}{|l|l|l|l|}
\hline & $\begin{array}{l}\text { Sedentario- } \\
\text { ligero }\end{array}$ & $\begin{array}{l}\text { esfuerzo } \\
\text { medio-alto }\end{array}$ & Total \\
\hline No IP & 16 & 9 & 25 \\
\hline Si IP & 58 & 17 & 75 \\
\hline Total & 74 & 26 & 100 \\
\hline
\end{tabular}

Chi $2=1.1088 \quad$ Chi2 2 in corregir $=1.7325$

Aunque los trabajadores calificados con una IPP no dejan su puesto de trabajo, la inclusión de estos casos en el grupo de "No IP" hubiera resultado intrascendente en nuestro estudio por su escaso número, no variando el grado de significación respecto del obtenido con la agrupación de variables expuesta.

\section{DISCUSIÓN}

De la comparación entre las variables "Alteración predominante en Ecocardiografía en reposo" e "Incapacidad Permanente", se deduce que NO existen diferencias significativas en las alteraciones ecocardiográficas en reposo entre los calificados como incapacitados y los no calificados como tales por el EVI.

Para ver si este resultado se debió a la afectación funcional preferente en los trabajadores de un tipo determinado respecto a otros, comparamos las variables "Grupo funcional estimado por el Médico Evaluador" y "Calificación de IP por el EVI", concluyendo que No existían diferencias significativas en el grado funcional estimado por el Médico Evaluador, entre los calificados como incapacitados por el EVI y los no calificados como tales (con la precaución de considerar la posibilidad de que la muestra no fuera suficientemente representativa por existir algunas celdas con menos de 5 casos).

Por tanto, ante tal homogeneidad (aparentemente discordante con el concepto de incapacidad en relación al grado funcional y a los datos objetivos de alteración cardiaca en ecocardiografía en reposo), comparamos las variables "Tipo de trabajo" y "Grado de IP". De dicha comparación concluimos que NO existe diferencia significativa en la distribución de la calificación de IP en los trabajadores dedicados a tareas sedentarias-ligeros esfuerzos respecto a los dedicados a tareas de esfuerzo físico medioalto.

Así, sobre el valor de los hallazgos ecocardiográficos aplicados en la calificación de la I.P. emitida, podría decirse que el mero diagnostico de cardiopatía se asoció a la calificación de incapacidad (laboral) permanente en un $75 \%$, con independencia del grado funcional y la actividad laboral desarrollada por el asegurado.

\section{CONCLUSIONES}

a. El grupo funcional estimado por el Médico Evaluador y las alteraciones en la función cardiaca detectadas por ecocardiografía en reposo NO pueden explicar la distribución de la calificación del grado de IP por el EVI, en la muestra estudiada.

La calificación, o no, como I.P. por el EVI, NO se relacionó con el nivel de esfuerzo genérico asignado a los puestos de los distintos trabajadores calificados; para una distribución similar, entre aquellos, del grado funcional estimado. Por ello, el tipo de trabajo NO puede justificar tampoco la distribución del grado de IP en la muestra estudiada, es decir, el diagnostico de cardiopatía se asoció a la calificación de incapacidad (laboral) permanente (en un 75\%), con independencia del grado funcional y la actividad laboral desarrollada por el asegurado.

b. No se disponía de datos sobre las alteraciones de la función cardiaca en el ejercicio, por no contar con pruebas de imagen bajo estrés - farmacológico o esfuerzo físico-, detectándose solo una frecuencia del $20 \%$ para estas alteraciones (hipo-disquinesias) en reposo.

c. Los parámetros aportados por la ecocardiografía en reposo no son suficientes para estimar el grado de repercusión funcional en los trabajadores evaluados. 


\section{SUGERENCIAS Y RECOMENDACIONES}

La medicina evaluadora aplicada a la incapacidad laboral, necesita patrones de valoración funcional con potencialidad pronóstica de la capacidad laboral, que mejoren el valor de los indicadores de utilidad diagnóstica que usamos actualmente.

Estimamos necesaria la puesta en marcha de estudios prospectivos para evaluar la capacidad funcional efectiva en trabajadores con y sin calificación de incapacidad laboral, para establecer el grado de relación con los resultados de las pruebas diagnósticas utilizadas en el procedimiento evaluador.

Consideramos necesario someter el método actualmente en uso (y con ello, a las escalas y pruebas complementarias más determinantes) al análisis coste-beneficio (ACB), como única forma de determinar la eficiencia de éstas en Medicina Evaluadora (a la hora de discriminar en la calificación de Incapacidad Laboral). No es objeto de este trabajo profundizar en ello, solamente plantear el escenario teórico de su necesidad.

Debe profundizarse en el estudio de los "criterios" y "parámetros clave" considerados por el Organo Colegiado calificador de la IP (EVI), para trabajar en ellos durante la elaboración del IMS y consensuar

\section{BIBLIOGRAFÍA}

1.- Ángel Arancon Vigueta. Teoría y Practica de la Medicina Evaluadora. FUNDACIÓN Mapfre MEDICINA; 2004.

2.- Begoña Bermejo Fraile. Epidemiología Clínica aplicada a la toma de decisiones en medicina. ANALES DEL SISTEMA SANITARIO DE NAVARRA;2003.

3.- Francesc Roca. Valoración del Daño Corporal. Evidencias para los litigios. 1997. Disponible en la World Wide Web :<http:// www. La-plaza.com/vdc >>

4.- Manual de Actuación para Médicos del INSS. Madrid. INSS; 2003.

5.- Real Decreto 1300/1995, por el que se desarrolla, en materia de incapacidades laborales del Sistema de Seguridad Social, la Ley 42/1994, de 30-12-1994. BOE de 19 agosto 1995.

6.- Organización Mundial de la Salud: Clasificación Internacional del Funcionamiento de la Discapacidad y de la Salud; 2001. su repercusión funcional objetiva con fundamento médico-científico.

Los requerimientos psico-físicos de la actividad laboral de cada trabajador deben precisarse con criterios objetivos, a fin de compararse con los índices de repercusión funcional utilizados por el EVI y la Unidad Médica que elabora los IMS.

Lo expuesto en este estudio sería aplicable a un numeroso grupo de pruebas complementarias (electrofisiológicas, de imagen, analíticas, etc.) cuya validez probada en clínica-diagnóstica no tiene validada su aplicación directa en la estimación de la incapacidad laboral.

Por todo ellos, se requieren más estudios para identificar los índices de función cardiaca que, en las diferentes patologías, aporten datos validos, sensibles y específicos que puedan ser utilizados en la valoración de la capacidad de esfuerzo físico "útil" de los trabajadores con enfermedades cardiacas. Igualmente, se requieren más estudios en el ámbito de la categorización de los puestos de trabajo, para conocer realmente los requerimientos físicos de éstos mediante indicadores fiables (que posibiliten su comparación con el grado funcional del trabajador estimado por el Médico Evaluador en los trabajadores con enfermedad cardiaca).

7.- Guía de valoración del menoscabo permanente Instituto Nacional de Medicina y Seguridad en el Trabajo. Segunda Edición; 1999.

8.- Valeriano Sosa Rodríguez. Estudio de la Incapacidad Laboral por Enfermedades Cardiocirculatorias. INMST. 2 2a Edición; 1998.

9.- XIX Diplomatura en Valoración Médica de Incapacidades. Escuela Nacional de Medicina del Trabajo (ISC III). Madrid; 2006/2007.

10.- Real Decreto Legislativo 1/1994 de 20.06.1994. Texto Refundido de la LGSS.

11.- Eva Laraudogoitia y Jose L. Moya. Actualización en técnicas de imagen cardiaca. Ecocardiografía y cardiorresonancia magnética. Rev.Esp.Cardiol 2006; 59:31-37.

12.- Ángel $\mathrm{M}^{\mathrm{a}}$ Alonso, Carlos Paré y col. Papel de la Ecocardiografía Doppler en el manejo de la cardiopatía isquémica crónica. Rev. Esp. Cardiol 1997; 50: 15-25 
13.- Juan Quiles, Miguel A. García-Fdez. y col. Ecocardiografía portátil: análisis comparativo de los resultados obtenidos frente a los estudios estándar. Rev. Esp. Cardiol 2003; 56: 480-486.

14.- Ricardo Ernesto Ronderos. Ventajas y desventajas de la ecocardiografía en la evaluación de la insuficiencia cardiaca. $1^{\circ}$ Congreso Virtual de Cardiología. Argentina.

15.- Hector Bueno Zamora. Ecocardiografía y Doppler cardiaco. H.U. Gregorio Marañon. Madrid.

16.- Guías para la Determinación de la Incapacidad Laboral en pacientes cardiacos. Sección de cardiología preventiva y rehabilitadora de la Sociedad española de Cardiología.

17.- Rodolfo Pizarro y Pablo Oberti. El año de la Ecocardiografía. Weyman AE. J. Am. Col. Cardiol 2004; 43:140-8.

18.- Almenares Pujadas, M.E., Berovides Padilla, O. y col. Evaluación ecocardiográfica en judokas olímpicos. Rev. Int. Med. Cienc. Act. Fis. Deporte- num 21- marzo 2006.

19.- Muñoz Mtnez, L. Utilidad del ecocardiograma en la valoración del paciente con insuficiencia cardiaca diastólica. Rev. Urug. Cardiol. V.20 n.2.2005.

20.- Señor, J.M., Escobar, C. y col. Sensibilidad y Especificidad de la ecocardiografía de estrés con dobutamina para la detección de la enfermedad coronaria. Rev. Colomb. Cardiol Ago 2001; 9 (1): 5-10.

21.- Centro Médico Caracas. Experiencia de la ecocardiografía de estrés en America Latina. Revisión de 5 años. Harry Acquatella. Venezuela. Rev. Esp. Cardiol 2003; 56 (supl 1): 21-8.

22.- Ella A. Kazaranooni, MD. ¿Qué modalidad de diagnóstico por imagen elegimos para cada paciente?
Hemodinamiadelsur.com, Cardiosource. Conversations with experts.

23.- Fortuño, M.A., Zalba, G., y col. Cardiopatía Hipertensiva. Vol. 21, Suplemento1. Unidad de fisiopatología Vascular, Facultad de Medicina, Universidad de Navarra.

24.- Victor, R., Balestrini, R., y col. Valor del Ventriculograma Isotópico de esfuerzo en la práctica clínica. $2^{\circ}$ Congreso Virtual de Cardiología. Servicio de Med. Nuclear, Instituto Modelo de Cardiología, Córdoba, Argentina.

25.- Conde Cerdeira, H. Y col. Manejo de la angina de pecho en el consultorio del Médico de familia. Rev. Cubana Med. Gen. Integr. Nov-dic-2000; V16 n16.

26.- Yu-Chen Chang, MD, Jung-Der Wang, MD. Cumulative injury rate and potential workdays and salary lost. Scand J. Work Environ Health 1995; 21: 494-503.

27.- J.Ilmarinen, G. Costa. L'invecchiamento dei lavoratori nell'unione europea. Clínica del Lavoro, Milano. Med Lav. 2000; 91,4: 279-295.

28.- Ph. Meunier. Pour une modelisation des profils cardiaques de postes. CAMIP-2000-4.

29.- Karen Belkic, Reza Emdad and Töres Theorell. Occupational profile and cardiac risk : possible mechanisms and implications for professional drivers. Int. J. of Occupational Medicine and Environmental Health 1998; Vol. 11, No 1, 37-57.

30.- Arana Munárriz, V., Echeverría Aizpuru, I. Monitorización de la frecuencia cardiaca en el estudio de la carga física en el trabajo. Mapfre SEGURIDAD. $N^{\circ} 73-1^{\circ}$ T- 1999.

31.- G. Costa. Capatitá di lavoro e invecchiamento. Med. Lav. 2000; 91, 4: 302-312. 